\title{
New distance estimates to RX Eri, an RR Lyrae star in Eridanis, using near infrared photometry
}

\author{
TIMOTHY JONES ${ }^{1, *}$ \\ ${ }^{1}$ Alfred Deakin High School, ACT Education Department \\ "Corresponding author: timdjones7@gmail.com
}

\begin{abstract}
This paper presents the results of analysis of the RRab type star, RX Eri, building on previous work to include light curves further into the infrared. This project has yielded light curve results which have determined the period to $0.587 \pm 0.005 \mathrm{~d}$, agreeing with prior research. The distance estimation using Period-Luminosity-Metallicity relationships, V: $612 \pm 27$ pc, i: $608 \pm 21$ pc, z: $613 \pm 23$ pc broadly agreed with Gaia DR2's estimation of $605 \pm 11 \mathrm{pc}$. These infrared filters yielded better estimations in terms of error than those in the $\mathrm{V}$ band, although they all fall within the margins of error of the measurements made.
\end{abstract}

(C)(1) $\odot \odot 2020$ Astronomy Theory, Observations and Methods Journal

Keywords: Stars: Variable: RR Lyrae, techniques: photometric, catalogs, parallaxes

https://doi.org/10.32374/atom.2020.1.2

\section{INTRODUCTION}

RR Lyrae stars are used as tools to determine the distance to star clusters in our galaxy which is made possible by photometric analysis of their light curves in a range of frequencies. This paper presents the results of one such analysis of the star, RX Eri, building on previous work to include light curves further into the Infra red. The use of RR Lyrae variable stars as standard candles for the measurement of distances and ages of globular clusters will be improved if the error can be reduced by using filters in the near infrared (Caceres \& Catelan, 2008).

Catelan et al (2004) demonstrated that more reliable distance calculations are possible when analysing RR Lyrae stars in the near infrared compared to traditional optical observations. This analysis showed that the Period-Luminosity relationship becomes more linear and tighter as the light frequency moves towards the near infrared ( $\mathrm{V}$ band $\mathrm{r}=0.19, \mathrm{~K}$ band $\mathrm{r}=0.9990$ ) (Catelan, Pritzl, \& Smith, 2004). This relationship has been shown to best suit the accurate determination of distance using absolute magnitudes and most suited to modelling the Period-Luminosity relationship in RR Lyrae stars (Caceres \& Catelan, 2008).

Observations of RX Eri demonstrate a reliable measurement of its period and class (RRab) across many authors (Carrillo et al., 1995; Kovacs, 2005; Neeley et al., 2017; Andrievsky et al., 2017; Siegel, 1982). A summary of RX Eri data is presented in table 1.

Metallicity measurements however are in stark contrast and and this represents a challenge to this study. As Table 2 shows, there has been a large amount of variability in the measurement of metallicity which is difficult to critique without detailed analysis of the various methods used. In the absence of this information the calculations used for this paper, the value determined by (Neeley et al., 2017) was selected as 
Table 1. RX Eri General Properties

\begin{tabular}{ccc}
\hline Type & Value & Source \\
\hline ICRS & Ra 44944.29 & (Gaia et al., 2018) \\
Coordinates & Dec -154431.8 & \\
Period & $0.587246 \mathrm{~d}$ & (Carrillo et al., 1995) \\
& $0.587247 \mathrm{~d}$ & (Kovacs, 2005) \\
& $0.587242 \mathrm{~d}$ & (Neeley et al., 2017) \\
Teff & $6178 \mathrm{~K}$ & (Andrievsky et al., 2017) \\
& $6300 \mathrm{~K}$ & (Siegel, 1982) \\
Radial & $80 \mathrm{Km} / \mathrm{s}-1$ & (Andrievsky et al., 2017) \\
Velocity & & \\
Spectral Type & F2D & (Gaia et al., 2018) \\
Mv & 0.41 & (Siegel, 1982) \\
& 0.66 & (Fernley, Carney, Skillen, Cacciari, \& Janes, 1998) \\
& 0.54 & (Woolley \& Dean, 1976) \\
Va/Fe & 9.72 & (Kovacs, 2005) \\
& 9.70 & (Carrillo et al., 1995) \\
Vmag & 9.69 & (Muraveva et al., 2015) \\
& 0.52 & (Andrievsky et al., 2017) \\
& &
\end{tabular}


it represented the measurement most related to the time the photometric measurements were made and is also a mode value of the data presented. Table 2. Measurement of Metallicity of Rx Eri
from past studies

\begin{tabular}{cc}
\hline $\mathrm{Fe} / \mathrm{H}$ & Source \\
\hline-1.07 & Kovacs 2005 \\
-1.18 & Andrievsky et al 2017 \\
-1.32 & Butler 1975 \\
-1.63 & Fernley et al 1997 \\
-1.33 & Neeley et al 2018 \\
-1.98 & Muraveva 2015 \\
-1.4 & Skillen et al 1992 \\
\hline
\end{tabular}

\section{METHODS}

\section{Observations}

The data upon which this project is based was gathered using the Las Cumbres Observatory (LCO) $0.4 \mathrm{~m}$ SBIG telescopes at Siding Springs, (Australia), Sutherland (South Africa), Cerra Tololo (Chile), Haleakala (Maui) and Teide (Spain). This experiment is dependant in the systems described in detail in Brown et al 2013 (Brown et al., 2013). The observational data, consisting of sets of $\mathrm{B}, \mathrm{V}, \mathrm{i}$ and $\mathrm{z}$ optical images was collected during a cadence of at least 70 observation opportunities as part of the Our Solar Siblings (Fitzgerald et al., 2018) proposal between February and April 2019.

Data gathered by the LCO telescope network was optimised to ensure that the images produced met the data collection needs but did not risk over-exposure, avoiding an unquantifiable error in the photometric measurements made. These optimal times were determined using AstroImageJ software ( AstroimageJ ) to count the pixels within an aperture and compare these to the sky background. In this manner the data collected represented the radiation created by only the target star. Optimal times for each band were determined to be 3 seconds for the $i$ band, 12 seconds for $\mathrm{z}$. 4 seconds for $\mathrm{V}$ and 13 seconds for $\mathrm{B}$ which represented $\approx 100000$ counts for $\mathrm{RX}$ Eri.

\section{Photometry}

The images and photometry were processed using the Our SolarSiblings pipeline (Fitzgerald, 2018). The SEK (Source Extractor Kron) files were found to produce the least variable photometry data and thus this method was used (Bertin \& Arnouts, 1996). This data was then further processed by astrosource (Astrosource). Astrosource identifies the most optimal stars in the image with the lowest variability to use as comparison star for each of the $\mathrm{B}, \mathrm{V}, \mathrm{i}$ and $\mathrm{z}$ filters used in this study. Astrosource analysis identified 6 standard stars, shown in Figure 1, with variability between 0.01-0.03 that were used as comparison sources. The known magnitudes are from APASS (Henden et al., 2016) for B and V and from Skymapper (Onken et al., 2019) for i and z. The comparison stars are listed in Table 3. These stars allowed the extraction of a calibrated lightcurve in each of these filters using differential photometry.

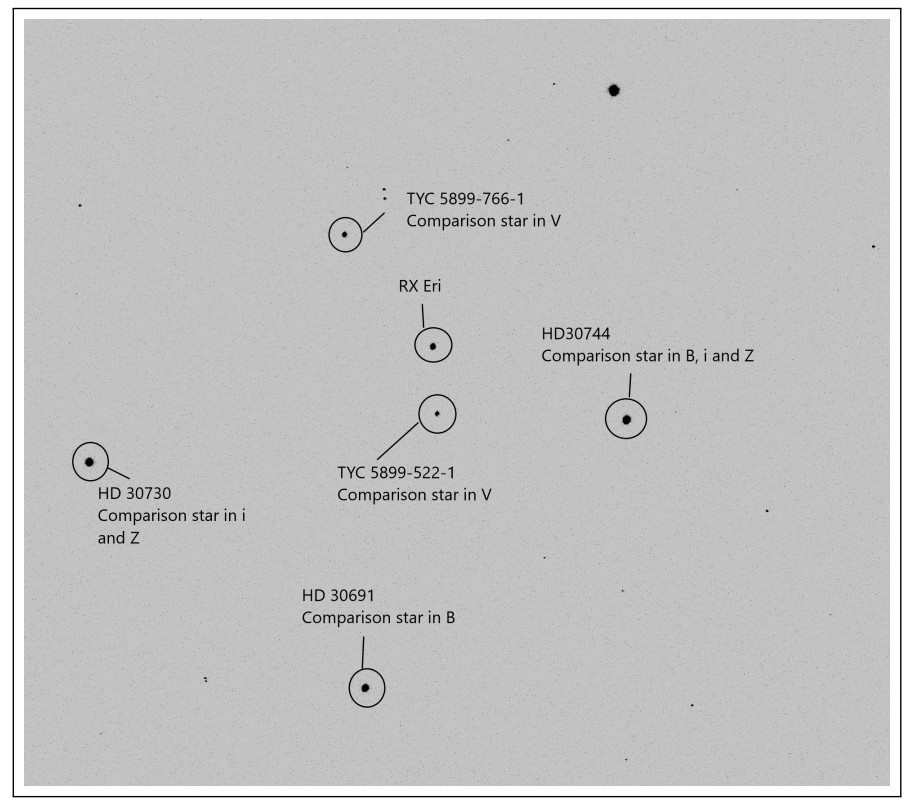

Fig. 1. Inverted image of the analysed starfield. Image taken with SBIG $0.4 \mathrm{~m}$ telescope operated by Las Cumbres Observatory

The final lightcurves were identified using the Phase Dispersion Method, coded by (Altunin, Caputo, \& Tock, 2020) to determine a period likelihood plot (see Figure 3) and folded on the determined period as shown below in figures 2-5.

In this paper we investigated the photometric properties of RX Eri using the robotic telescopes of the Las Cumbres Observatory network. Filter bands B,V, $\mathrm{i}$, and $\mathrm{z}$ were captured using the $0.4 \mathrm{~m}$ SBIG tele- 
Table 3. Calibration Stars Used

\begin{tabular}{ccccc}
\hline RA & Dec & Filter & Var & Mag \\
\hline 72.30953 & -15.7178 & B & 0.013 & 9.25 \\
72.40799 & -15.8093 & B & 0.030 & 8.77 \\
& & i & 0.012 & 7.90 \\
& & z & 0.014 & 8.16 \\
72.39154 & -15.6205 & i & 0.012 & 8.16 \\
72.40968 & -15.7428 & V & 0.017 & 10.99 \\
72.47515 & -15.7098 & V & 0.023 & 10.64 \\
72.39157 & -15.6205 & $\mathrm{z}$ & 0.010 & 8.24 \\
\hline
\end{tabular}

scopes of this network, allowing determination of light curves and an estimation of the distance to RX Eri.

\section{RESULTS}

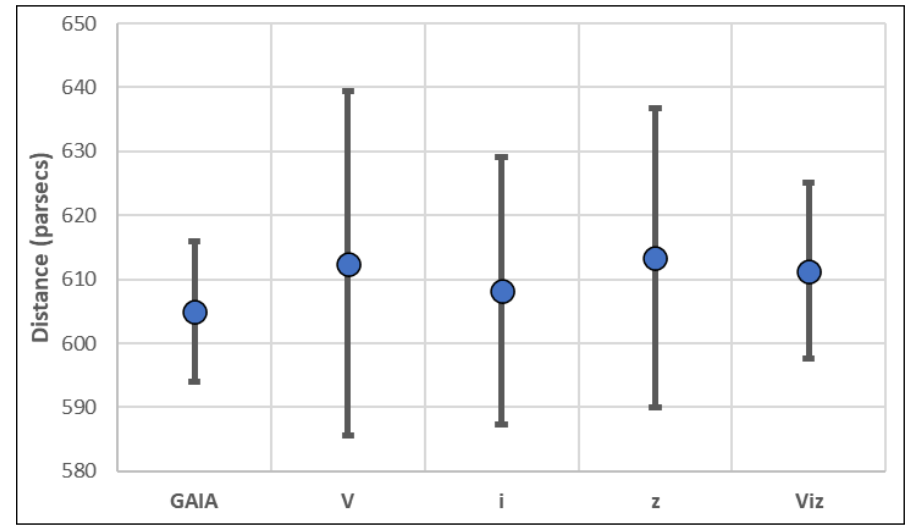

Fig. 2. Visual comparison of distance results from this study compared to GAIA

Distance estimation equations rely on the complex interactions of the chemical compositions of RR Lyrae stars as well as their period of flux and luminosity. Following the derived equations of (Bertelli, Bressan, Chiosi, Fagotto, \& Nasi, 1994) which account for the chemical composition of the target star in it's estimation of $\log (\mathrm{Z})$. The equation; $\log (Z)=$ $0.977 \mathrm{Fe} / \mathrm{H}-1.699$ was used to determine the $\log (\mathrm{Z})$ value for use in the distance calculations. Following this calculation the value was incorporated into the equations of period-luminosity-metallicity (Catelan et al., 2004; Caceres \& Catelan, 2008) used to determine the absolute magnitude and, hence, the distance to RX Eri as estimated by each band:

$$
\begin{gathered}
M v=2.288+0.882 \log Z+0.108(\log Z)^{2} \\
M i=0.908-1.035 \log P+0.220 \log Z \\
M z=0.839-1.295 \log P+0.211 \log Z
\end{gathered}
$$

Incorporating the distance modulus $m_{M}=5 \log d-$ 5 the distance (d) was determined for each band analysed. The interstellar reddening, $E(B-V) \approx$ 0.011 was estimated by minimising the variance in the distance across the three filters used. This value was reasonable given the maximum reddening in this direction of $E(B-V) \approx 0.05$ (Schlafly \& Finkbeiner, 2011). Distance errors were incorporated the errors in magnitude, period, metallicity.

The distance estimations using Period-LuminosityMetallicity relationships broadly agreed with Gaia DR2's (Gaia et al., 2018) estimation of $605 \pm 11$ pc. The distances are presented in Table 4 and shown visually in Figure 2. The combined estimate of the distance using all three of the V, i and z PLM estimates is $611 \pm 14 \mathrm{pc}$.

\section{Table 4. Distance Calculations}

\begin{tabular}{ccccc}
\hline Filter & $\mathrm{m}$ & $\begin{array}{c}\mathrm{M} \\
(\mathrm{pc})\end{array}$ & $\begin{array}{c}\text { Distance } \\
(\mathrm{pc})\end{array}$ & Error \\
\hline B & $10.148 \pm 0.015$ & & & \\
V & $10.104 \pm 0.021$ & 0.604 & 612 & 27 \\
i & $9.422 \pm 0.015$ & 0.476 & 608 & 21 \\
z & $9.452 \pm 0.014$ & 0.497 & 613 & 23 \\
\hline
\end{tabular}

Light curves were obtained in each filter which show a clear adherence to an RRab magnitude variation pattern. Comparing the $\mathrm{V}$ band results gained to AAVSO data (see figure 8) it is clear to see that the data gained for this study compares well to that of other observers.

\section{CONCLUSION}

This research has determined the period of RR Lyrae variable star, RX Eri to be 0.587 days, supporting the research of several other groups (e.g (Carrillo et al., 1995; Kovacs, 2005; Neeley et al., 2017). V band light curves comparable to past research have been generated and new observations in the near infrared range (bands $\mathrm{i}$ and $\mathrm{z}$ ) have been presented. The 
Research Article

Vol. 1, No. 1 / June 2020 / Astronomy Theory, Observations and Methods Journal 20

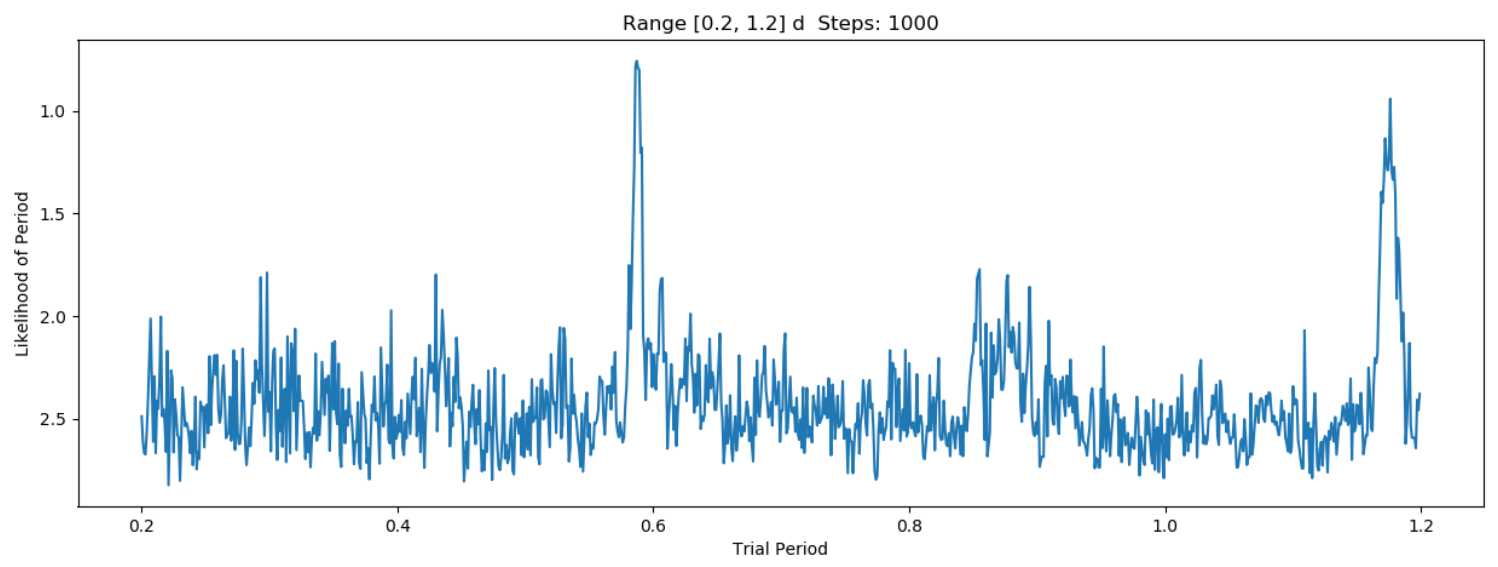

Fig. 3. PDM Likelihood plot in i band

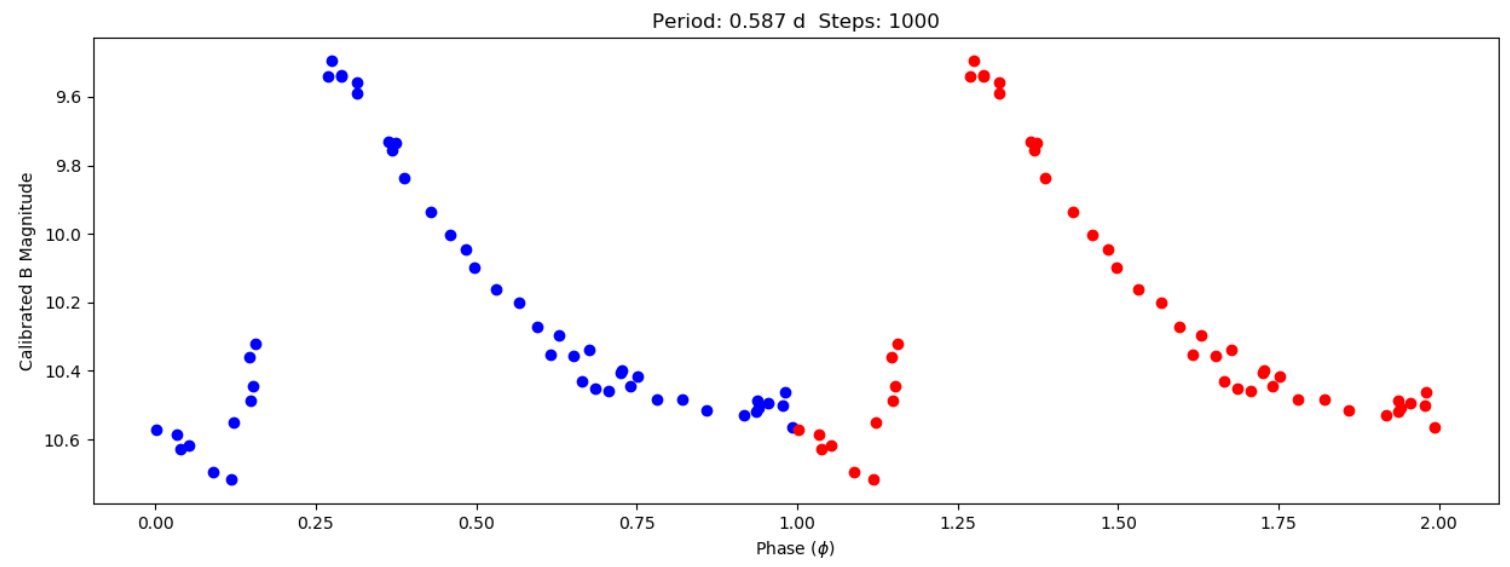

Fig. 4. Light curve in B (890 Angstroms)

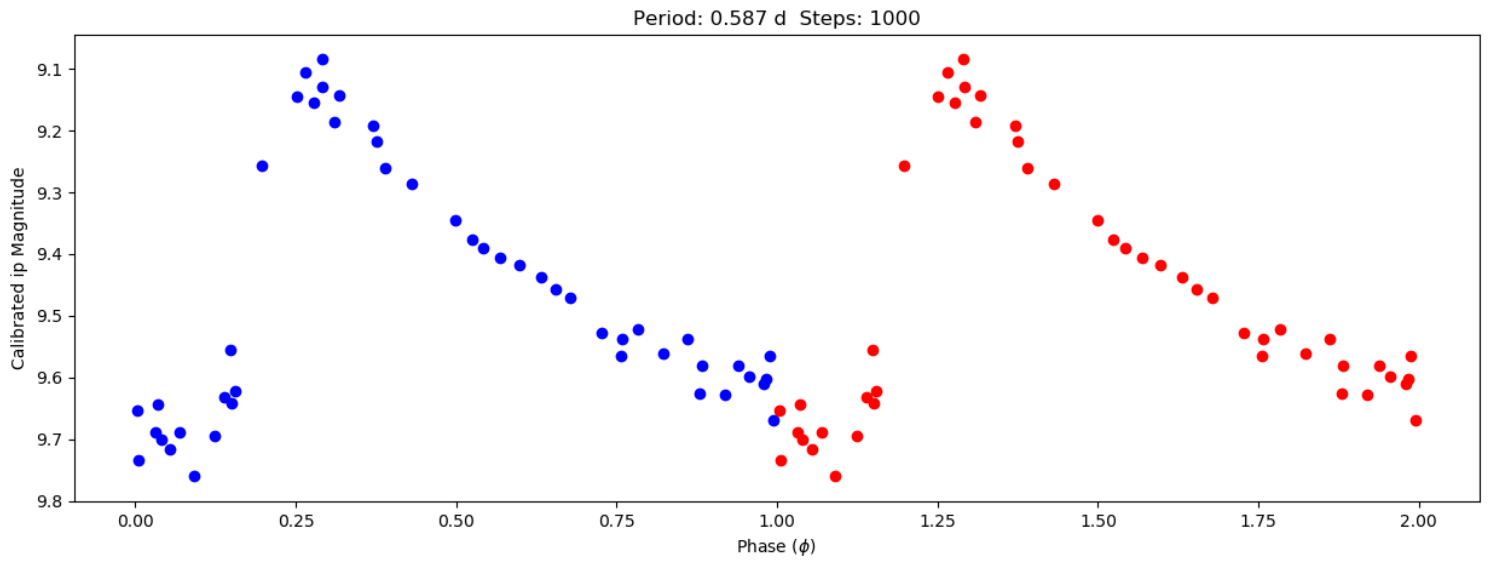

Fig. 5. Light curve in i (1290 Angstroms) 


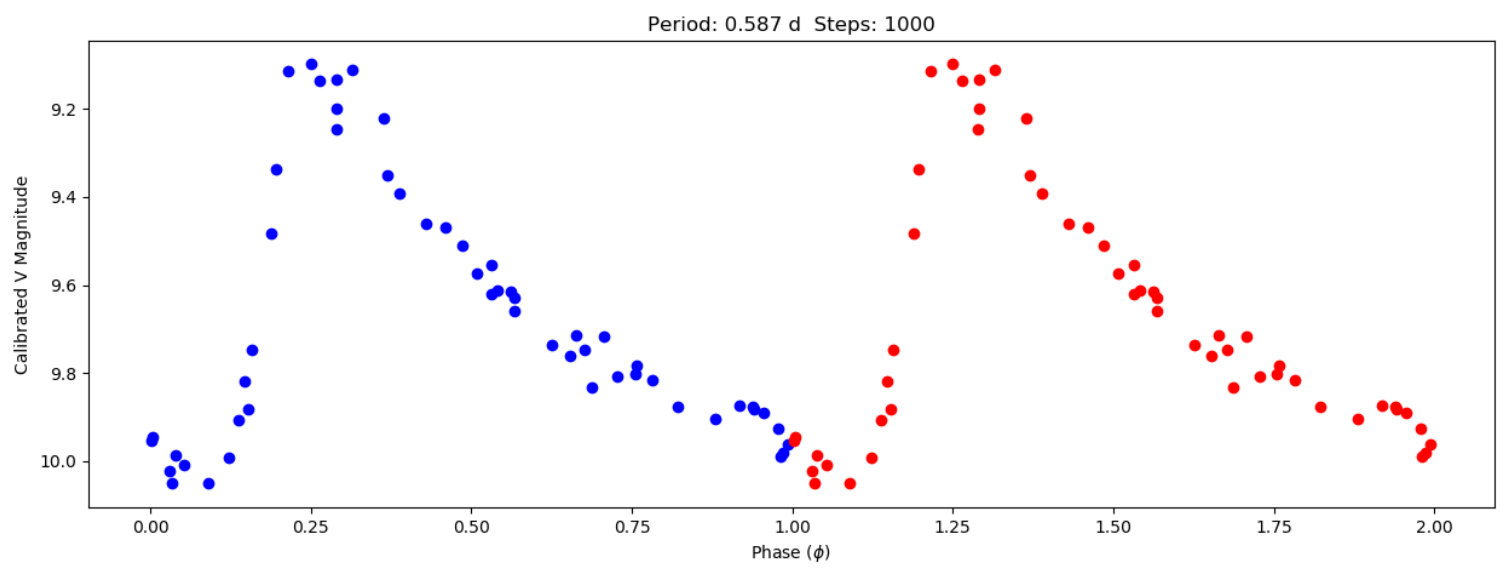

Fig. 6. Light Curve in V (840 Angstroms)

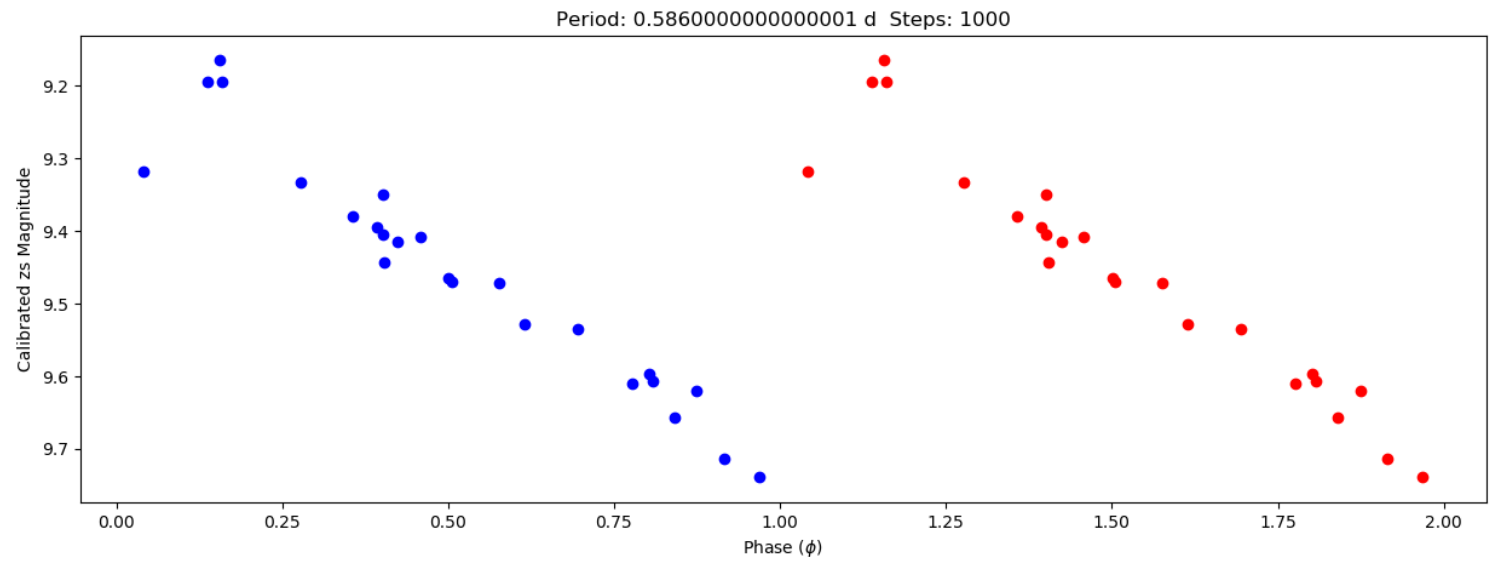

Fig. 7. Light Curve in z (1040 Angstroms)

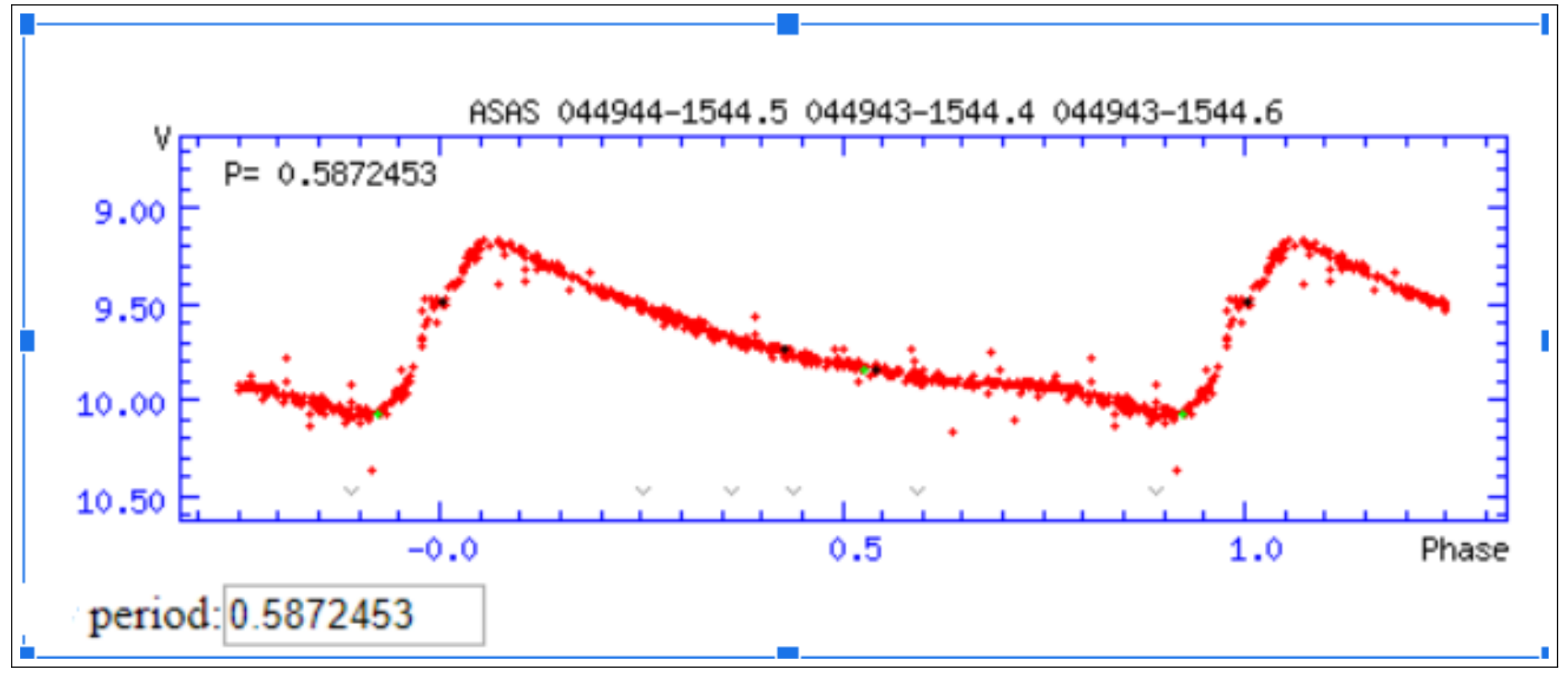

Fig. 8. Comparable V band data from AAVSO. After AAVSO 2005-19 accessed at AAVSO on 31.10.19 
distance estimations, V: $612 \pm 27 \mathrm{pc}, \mathrm{i}: 608 \pm 21 \mathrm{pc}$, z: $613 \pm 23$ pc. and Viz: $611 \pm 14$ pc. using the PeriodMetallicity-Luminosity relationships all agreed within the errors with Gaia DR2's estimation of $605 \pm 11$ pc.

\section{ACKNOWLEDGMENTS}

I would like to thank the continued support of the Our Solar Siblings staff, in particular Michael Fitzgerald, Alfred Deakin High School and the ACT Education Directorate for their support with this project. This work makes use of observations from the Las Cumbres Observatory Global Telescope Network without which the observations would not be possible. This research has made use of the International Variable Star Index (VSX) database, operated at AAVSO, Cambridge, Massachusetts, USA.

\section{REFERENCES}

Altunin, I., Caputo, R., \& Tock, K. (2020). Period of eclipsing binary epic 201458798. Astronomy Theory, Observations and Methods, 1(1).

Andrievsky, S., Wallerstein, G., Korotin, S., Lyashko, D., Kovtyukh, V., Tsymbal, V., ... Farrell, E. (2017). The relationship of sodium and oxygen in galactic field rr lyrae stars. Publications of the Astronomical Society of the Pacific, 130(984), 024201.

Bertelli, G., Bressan, A., Chiosi, C., Fagotto, F., \& Nasi, E. (1994). Theoretical isochrones from models with new radiative opacities. Astronomy and Astrophysics Supplement Series, 106, 275-302.

Bertin, E., \& Arnouts, S. (1996). Sextractor: Software for source extraction. Astronomy and Astrophysics Supplement Series, 117(2), 393-404.

Brown, T., Baliber, N., Bianco, F., Bowman, M., Burleson, B., Conway, P., ... others (2013). Las cumbres observatory global telescope network. Publications of the Astronomical Society of the Pacific, 125(931), 1031.

Caceres, C., \& Catelan, M. (2008). The periodluminosity relation of $\mathrm{rr}$ lyrae stars in the sdss photometric system. The Astrophysical Journal Supplement Series, 179(1), 242.

Carrillo, D., Burki, G., Mayor, M., Burnet, M., Lampens, P., \& Nicolet, B. (1995). Vizier online data catalog: Photometric and radial velocity of rrlyr stars (carrillo+ 1995). VizieR Online Data Catalog, 411.

Catelan, M., Pritzl, B. J., \& Smith, H. A. (2004). The rr lyrae period-luminosity relation. i. the- oretical calibration. The Astrophysical Journal Supplement Series, 154(2), 633.

Fernley, J., Carney, B., Skillen, I., Cacciari, C., \& Janes, K. (1998). The slope of the rr lyrae mv-[fe/h] relation. Monthly Notices of the Royal Astronomical Society, 293(3), L61-L64.

Fitzgerald, M. T. (2018). The our solar siblings pipeline: Tackling the data issues of the scaling problem for robotic telescope based astronomy education projects. RTSRE, 1(1), 347-358.

Fitzgerald, M. T., McKinnon, D. H., Danaia, L., Cutts, R., Salimpour, S., \& Sacchi, M. (2018). Our solar siblings. a high school focussed robotic telescope-based astronomy education project. RTSRE, 1(1), 221-235.

Gaia, C., Brown, A., Vallenari, A., Prusti, T., de Bruijne, J., Babusiaux, C., ... others (2018). Gaia data release 2 summary of the contents and survey properties. Astronomy \& Astrophysics, 616(1).

Henden, A. A., Templeton, M., Terrell, D., Smith, T., Levine, S., \& Welch, D. (2016). Vizier online data catalog: Aavso photometric all sky survey (apass) dr9 (henden+, 2016). yCat, II-336.

Kovacs, G. (2005). Iron abundances derived from rr lyrae light curves and low-dispersion spectroscopy. Astronomy \& Astrophysics, 438(1), 227-238.

Muraveva, T., Palmer, M., Clementini, G., Luri, X., Cioni, M.-R., Moretti, M. I., ... Rubele, S. (2015). New near-infrared period-luminositymetallicity relations for rr lyrae stars and the outlook for gaia. The Astrophysical Journal, 807(2), 127.

Neeley, J. R., Marengo, M., Bono, G., Braga, V. F., Dall'Ora, M., Magurno, D., ... others (2017). On a new theoretical framework for rr lyrae stars. ii. mid-infrared period-luminositymetallicity relations. The Astrophysical Journal, 841(2), 84.

Onken, C. A., Wolf, C., Bessell, M. S., Chang, S.W., Da Costa, G. S., Luvaul, L. C., ... Shao, L. (2019). Skymapper southern survey: Second data release (dr2). Publications of the Astronomical Society of Australia, 36.

Schlafly, E. F., \& Finkbeiner, D. P. (2011). Measuring reddening with sloan digital sky survey stellar spectra and recalibrating sfd. The Astrophysical Journal, 737(2), 103.

Siegel, M. J. (1982). A wbyb photometric study of 
rr lyr stars. i. Publications of the Astronomical Society of the Pacific, 94(557), 122.

Woolley, R., \& Dean, J. (1976). The radius of rx eridani. Monthly Notices of the Royal Astronomical Society, 177(1), 247-255. 